\title{
Implicit Medial Representation for Vessel Segmentation
}

\author{
Guillaume Pizaine $^{a b}$, Elsa Angelini ${ }^{b},{\text { Isabelle } \text { Bloch }^{b}, \text { Sherif Makram-Ebeid }}^{a}$ \\ ${ }^{a}$ Medisys Research Lab, Philips Healthcare, Suresnes, France. \\ ${ }^{b}$ Institut Telecom, Telecom ParisTech, CNRS LTCI, Paris, France.
}

\begin{abstract}
In the context of mathematical modeling of complex vessel tree structures with deformable models, we present a novel level set formulation to evolve both the vessel surface and its centerline. The implicit function is computed as the convolution of a geometric primitive, representing the centerline, with localized kernels of continuously-varying scales allowing accurate estimation of the vessel width. The centerline itself is derived as the characteristic function of an underlying signed medialness function, to enforce a tubular shape for the segmented object, and evolves under shape and medialness constraints. Given a set of initial medial loci and radii, this representation first allows for simultaneous recovery of the vessels centerlines and radii, thus enabling surface reconstruction. Secondly, due to the topological adaptivity of the level set segmentation setting, it can handle tree-like structures and bifurcations without additional junction detection schemes nor user inputs. We discuss the shape parameters involved, their tuning and their influence on the control of the segmented shapes, and we present some segmentation results on synthetic images, 2D angiographies, 3D rotational angiographies and 3D-CT scans.
\end{abstract}

Keywords: vessel segmentation, tree-like structures, level sets, variational methods, shape constraint

\section{INTRODUCTION}

Vascular tree segmentation is a challenging task for fully automated approaches and remains one of the most studied problems in medical imaging. Various dedicated image processing algorithms have been proposed for vessel enhancement, ${ }^{1}$ model-based segmentation, ${ }^{2,3}$ explicit or implicit active contour segmentation, ${ }^{4,5}$ or stochastic tracking $;{ }^{6,7}$ we refer the reader to Ref. 8 for an extensive survey on vessel modeling and segmentation. Segmentation algorithms usually extract the vessel boundary and recover the centerline of the vessels during two separate stages. Few research works ${ }^{9,10}$ have attempted to address the problem of extracting both simultaneously.

As an alternative, convolution surfaces, first introduced in the field of computer graphics and previously used for the visualization of tubular structures ${ }^{11}$ can be used. They are defined as the convolution of a shape primitive with a set of localized kernels and provide an implicit shape formulation similar to the envelop of spheres representation. ${ }^{10}$ In a recent work, ${ }^{12}$ a single branch vessel model based on convolution surfaces was introduced. However, the explicit parameterization of the centerline hindered its extension to multi-branch vessel trees. In this paper, we propose to reformulate the problem with the use of an implicit representation for the centerline. In $\mathrm{nD}$, the level set framework enables to define manifolds of co-dimension 1 (with one equation), corresponding to closed surfaces in $3 \mathrm{D}$ and closed contours in $2 \mathrm{D}$. Lorigo et al. ${ }^{4}$ proposed to extract vessel structures via the localization and regularization of its centerlines $\Gamma$ only, which defined manifolds of co-dimension 2 in 3D. In practice, the vessel centerline was defined as the epsilon-isolevel of a function $\Phi, \Gamma_{\epsilon}=\{\boldsymbol{x} \mid \Phi(\boldsymbol{x})=\epsilon\}$, where $\Phi$ was the signed distance function to a curve $\mathcal{C}$ and $\epsilon$ a real positive number that must be chosen as small as possible. Thus, $\Gamma_{\epsilon}$ was actually a thin tube around $\mathcal{C}$ being manipulated instead of $\mathcal{C}$.

Similarly to Ref. 4, our first contribution consists in modeling the vessel centerline as the epsilon-isolevel of an implicit function (Sect. 2.1). Our second contribution is a novel dedicated geometrical constraint designed to maintain the tubular shape of the vessels (Sect. 2.2). Finally, experimental results on synthetic and real image data are presented in Sect. 3.

Medical Imaging 2011: Image Processing, edited by Benoit M. Dawant, David R. Haynor, Proc. of SPIE Vol. 7962, 79623Q - ( 2011 SPIE · CCC code: 1605-7422/11/\$18 - doi: 10.1117/12.878048 


\section{IMPLICIT VESSEL REPRESENTATION WITH AN IMPLICIT MEDIAL AXIS}

\subsection{Description of the centerline model}

Lefevre et al. ${ }^{12}$ introduced the use of convolution surfaces to model tubular structures as implicit generalized cylinders. Assuming circular cross-sections, the vessel shape was encoded with an open parameterized centerline $\boldsymbol{m}(s):[0,1] \rightarrow \mathbb{R}^{n}$ convolved with a set of pointwise localized kernels with continuously varying scales $\sigma(s)$. The corresponding two-parameters level set function was defined as:

$$
\Phi_{\boldsymbol{m}, \sigma}(\boldsymbol{x})=\int_{0}^{1} \omega\left(\frac{\|\boldsymbol{x}-\boldsymbol{m}(s)\|}{\sigma(s)}\right)\left\|\boldsymbol{m}^{\prime}(s)\right\| \mathrm{d} s-C,
$$

where $C$ was an arbitrary positive constant used to force negative values outside the vessels, and $\omega$ was a nonnormalized Gaussian kernel $\omega(\boldsymbol{x})=\exp \left(-k \boldsymbol{x}^{2}\right), k \in \mathbb{R}^{+}$. The vessel segmentation problem was then formulated as an optimal two-phase partition problem of the image domain $\Omega$ of an image $I$, in which foreground (first phase) and background (second phase) corresponded to the vessel tree and the surrounding structures, respectively. Computing the intensity distributions $p_{i}, i=1,2$, of the foreground and the background, both regions were described by the homogeneity measures $r_{i}(\boldsymbol{x})=-\log p_{i}(I(\boldsymbol{x})),{ }^{13}$ and the following functional $E$ was minimized over the set of all possible image partitions identifying the first phase as a region $\mathcal{A}$ of $\Omega$ :

$$
E=\mathcal{R}(\mathcal{A})+\int_{\mathcal{A}} r_{1}(\boldsymbol{x}) \mathrm{d} \boldsymbol{x}+\int_{\Omega \backslash \mathcal{A}} r_{2}(\boldsymbol{x}) \mathrm{d} \boldsymbol{x} .
$$

where $\mathcal{R}(\mathcal{A})$ was a regularization term which enforces shape regularity.

We propose an alternative implicit formulation of the centerline to allow for natural evolutions of tree-like shapes into branches. The vessel boundaries are still modeled as the zero-level set of a function $\Phi$ expressed as a convolution surface, but the primitive is now the characteristic (Heaviside) function of a medialness function $h: \mathbb{R}^{n} \rightarrow \mathbb{R}$ (Fig. 1):

$$
\Phi_{h, \sigma}(\boldsymbol{x})=\int_{\Omega} H(h(\boldsymbol{y})) \omega\left(\frac{\|\boldsymbol{x}-\boldsymbol{y}\|}{\sigma(\boldsymbol{y})}\right) \mathrm{d} \boldsymbol{y}-C .
$$

The centerline of a vessel is represented as a thin tubular volume defined as $\boldsymbol{x} \mid h(\boldsymbol{x})>0$. In a discrete setting, the extent of this volume should be approximately the image resolution to avoid the risk that many kernels may contribute to a single point on the vessel surface. Thus, we are able to identify the scale parameter $\sigma$ with the vessel radii and to define the vessel surface as the zero-level set of $\Phi_{h, \sigma}$.

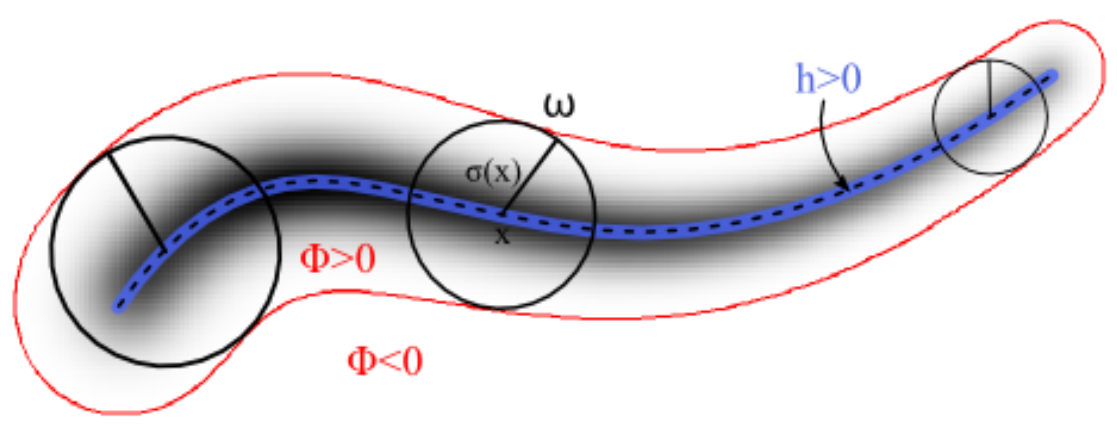

Figure 1. Implicit representation of a tubular structure in 2D. The foreground, in red, is modeled as the convolution of a blue thin area $(h>0)$ localizing the vessel centerline with local kernels $\omega$ of radius $\sigma$.

This new formulation leads to the following reformulation of the energy to be minimized (2):

$$
E_{h, \sigma}=\mathcal{R}(h, \sigma)+\int_{\Omega} H\left(\Phi_{h, \sigma}(\boldsymbol{x})\right) r(\boldsymbol{x}) \mathrm{d} \boldsymbol{x},
$$


where $r(\boldsymbol{x})=r_{1}(\boldsymbol{x})-r_{2}(\boldsymbol{x})$. In practice, $C$ is set based on the assumption that on a straight cylinder, a point on the surface is generated by the contribution of only one kernel. Using standard calculus of variations and the generalized scaling property of the Dirac distribution, we derive the following gradient-descent scheme for $h$ and $\sigma:$

$$
\begin{gathered}
\nabla_{h} E(\boldsymbol{y})=\delta(h(\boldsymbol{y})) \int_{\{\Phi=0\}} \frac{r(\boldsymbol{x})}{\|\nabla \Phi(\boldsymbol{x})\|} \omega\left(\frac{\|\boldsymbol{x}-\boldsymbol{y}\|}{\sigma(\boldsymbol{y})}\right) \mathrm{d} \boldsymbol{x}, \\
\nabla_{\sigma} E(\boldsymbol{y})=-H(h(\boldsymbol{y})) \int_{\{\Phi=0\}} \frac{r(\boldsymbol{x})}{\|\nabla \Phi(\boldsymbol{x})\|} \frac{\|\boldsymbol{x}-\boldsymbol{y}\|}{\sigma(\boldsymbol{y})^{2}} \omega^{\prime}\left(\frac{\|\boldsymbol{x}-\boldsymbol{y}\|}{\sigma(\boldsymbol{y})}\right) \mathrm{d} \boldsymbol{x} .
\end{gathered}
$$

\subsection{Coupled constraints on medialness and scales}

Since the geometry of the vessel is encoded with continuous spatial variables having smooth variations within the vessel and strong gradients at the vessel interface, we penalize the Total Variation norm of $h$ and $\sigma:{ }^{14}$

$$
\mathcal{R}(h, \sigma)=\lambda \int_{\Omega}\|\nabla h(\boldsymbol{x})\| \mathrm{d} \boldsymbol{x}+\mu \int_{\Omega}\|\nabla \sigma(\boldsymbol{x})\| \mathrm{d} \boldsymbol{x}, \quad \lambda, \mu \in \mathbb{R} .
$$

Our initial experiments showed the need for additional constraints to prevent the medialness function $h$ from spreading inside the objects to be segmented.

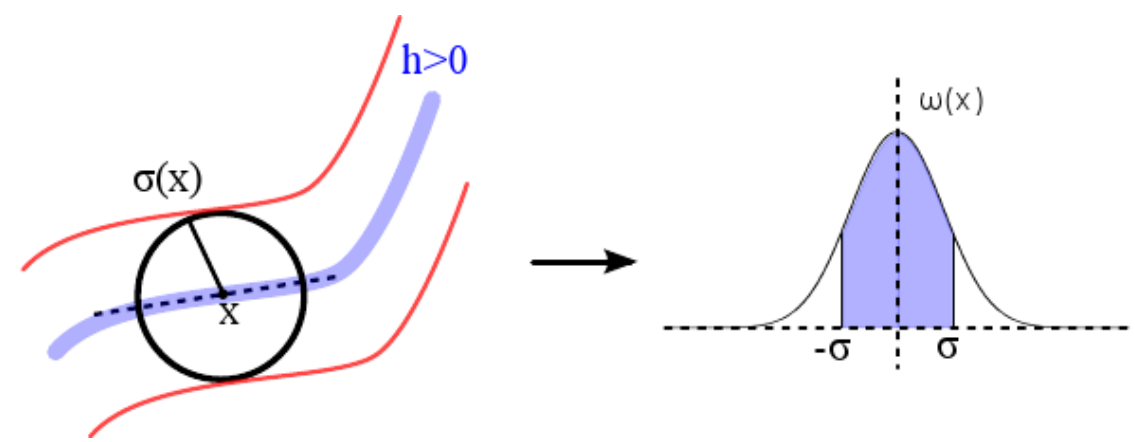

Figure 2. The volume of the centerline lying inside a local kernel centered at $\boldsymbol{x}$ (on the left) is close to the volume of the blue-shaded area under the Gaussian profile (on the right). The size of the kernel can be locally adapted to the vessel geometry, or can be set constant for simplicity.

In Ref. 15, Nain et al. introduced a simple yet effective volume constraint for a level set-based vessel segmentation framework. Within our framework, we are able to define a more restrictive constraint by measuring the volume of $h$, instead of $\Phi$, inside a neighborhood of locally-adapted size defined by $\sigma$ :

$$
V(\boldsymbol{x})=\int_{\Omega} H(h(\boldsymbol{y})) \omega\left(\frac{\|\boldsymbol{x}-\boldsymbol{y}\|}{\sigma(\boldsymbol{x})}\right) \mathrm{d} \boldsymbol{y} .
$$

Given a location $\boldsymbol{x}$ where $h(\boldsymbol{x})>0, h$ will be tubular around $\boldsymbol{x}$ if the corresponding local Gaussian kernel $\omega$ encompasses a volume of $h$ close to the volume of a straight tube going through its center. This equivalent volume can be expressed analytically as a function $V_{\sigma}$ of $\sigma(\boldsymbol{x})$ (Fig. 2). In 2D, the expected volume is typically:

$$
V_{\sigma}(\boldsymbol{x})=\sigma(\boldsymbol{x}) \sqrt{\frac{\pi}{k}} \operatorname{erf}(\sqrt{k})
$$

where erf is the error function and $k$ is the constant parameter of the Gaussian kernel (see Sect. 2.1). Penalizing deviations from this expected value, we propose a novel volume constraint $E_{\mathrm{v}}$ as:

$$
E_{\mathrm{v}}=\frac{1}{2} \int_{\Omega}\left(V(\boldsymbol{x})-V_{\sigma}(\boldsymbol{x})\right)^{2} \mathrm{~d} \boldsymbol{x}
$$


In practice, the constraint is simplified by fixing $\sigma$ to a reasonable value so that the hypothesis previously stated still holds. The volume constraint can thus be computed efficiently, in a single pass, as a convolution of the Heaviside of the medialness function $h$.

Finally, the evolution of the medialness function is driven by a third additional constraint $E_{m}$ that ensures local alignment of its gradient with the locally smoothed and normalized gradient field of the image $\boldsymbol{n}_{\sigma}(\boldsymbol{x})=\nabla_{\sigma(\boldsymbol{x})} I(\boldsymbol{x}) /\left\|\nabla_{\sigma(\boldsymbol{x})} I(\boldsymbol{x})\right\|:$

$$
E_{m}=\frac{1}{2} \int_{\Omega}\left\|\nabla h(\boldsymbol{x})-\boldsymbol{n}_{\sigma}(\boldsymbol{x})\right\|^{2} \mathrm{~d} \boldsymbol{x} .
$$

This constraint quantifies the local asymmetry of the image gradients. Smoothing normalized gradients allows to identify basins where strong and aligned gradients prevail, and the boundaries between two such basins correspond to the locations of medial structures such as centerlines.
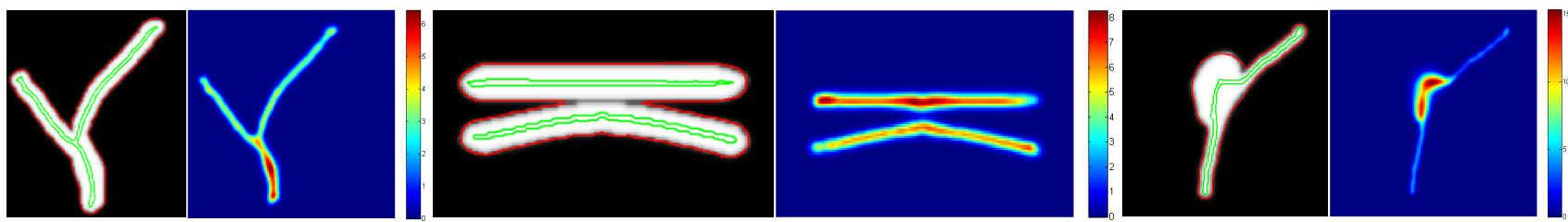

Figure 3. Segmentation results on synthetic images showing the initial image with the segmentation (red) and the centerline (green) overlaid, and the corresponding radius map. A piecewise-constant intensity model was used. From left to right: a Y-junction, tangent vessels and an aneurysm. Note that although tangent vessels have a single outside contour, their centerlines remain well-separated.

\section{EXPERIMENTAL RESULTS AND DISCUSSION}

In this section, we illustrate the performance of the proposed segmentation framework on 2D synthetic images (Fig. 3) and 2D and 3D angiographies of various anatomical regions (Fig. 4, 5). For well-contrasted images (including synthetic images), the initialization of the evolution process consists of a single click inside the object and a corresponding estimation of the radius. However, medical images require a more accurate initialization in order to compute the initial region models accurately.

\subsection{Model initialization with gradient diffusion}

For this purpose, we used an approach based on the Gradient Vector Flow (GVF $)^{16}$ similar to the idea developed by Bauer and Bischof. ${ }^{17}$ The GVF of an image $I$ is defined as the vector field $\boldsymbol{U}$ minimizing

$$
\int_{\Omega} \alpha\|\nabla \boldsymbol{U}\|^{2}+\|\nabla f\|^{2}\|\boldsymbol{U}-\nabla f\|^{2} \mathrm{~d} \boldsymbol{x}
$$

where $\alpha \in \mathbb{R}^{+}$and $f$ is chosen such that the vector field $\nabla f$ points towards the expected structures. In Fig.4, vessels are surrounded by a brighter background, so $f=-I$. On the contrary, adjacent tissues are darker than the vessels in Fig.5, so $f=I$.

To recover the medialness information, strong gradients in the image are diffused in uniform regions: the second term in Eq.12 enforces data fidelity where gradients are strong, whereas the first term smoothes the vector field where no information exists. The divergence of the resulting normalized vector field yields a map where high values characterize discontinuous orientations of the vector field, whereas small values identify regions with homogeneous directions. Strong discontinuities occur where strong gradients, originally from opposite edges, are present. This enables to identify the centerlines of the structures as a subset of the ridges of the map.

The centerlines are then recovered during a height ridge traversal step. ${ }^{18}$ The response of the divergence operator is weaker at bifurcations, so seeds $\left\{\boldsymbol{s}_{i}\right\}$ are selected as the local maxima of the divergence map having image intensity values $I\left(\boldsymbol{s}_{i}\right)$ above (or below) a given threshold. In our experiments, this threshold was set as the image median value. Given a voxel $\boldsymbol{x}_{\boldsymbol{i}}^{\mathbf{0}}=\boldsymbol{s}_{i}$, we first compute an estimation of the local orientation $\boldsymbol{t}_{i}^{0}$ at seed 
points using the second derivatives of the map. Considering the neighbors $\left\{\boldsymbol{x}_{n}\right\}$ of $\boldsymbol{x}_{\boldsymbol{i}}^{\mathbf{0}}$ such that $\boldsymbol{t}_{\boldsymbol{i}}^{\mathbf{0}} \cdot \boldsymbol{x}_{\boldsymbol{i}}^{\mathbf{0}} \boldsymbol{x}_{\boldsymbol{n}}>\beta$, $\beta \in[-1 ; 1]$, the point $\boldsymbol{x}_{n}$ providing the highest value is selected as the next point $x_{i}^{1}$ of the ridge. This selection continues until a point that has already been traversed is encountered, or until the map value decreases below a given threshold. The procedure is repeated for the same seed in the opposite direction $-t_{i}^{0}$, then for each seed $s_{i}$. Finally, the local radius is estimated by following the GVF vector field from a point on a ridge to the first local gradient extremum.

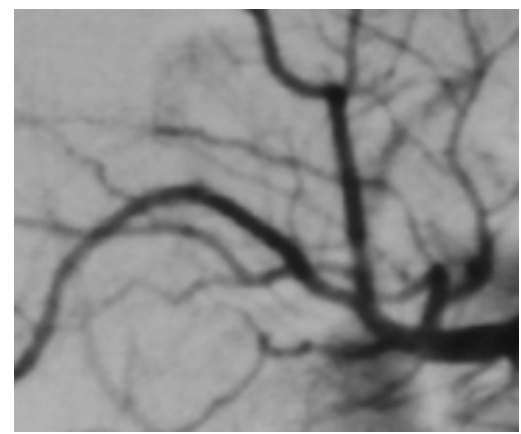

(a)

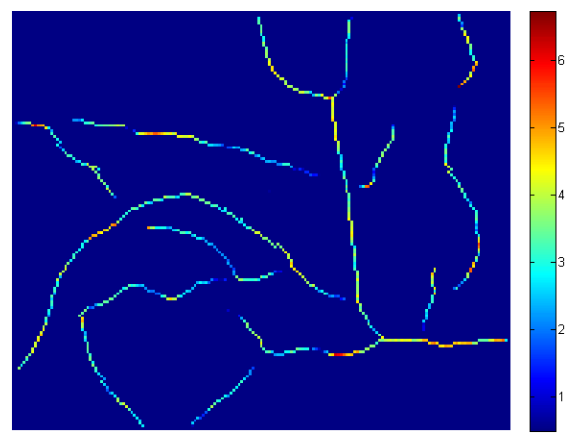

(b)

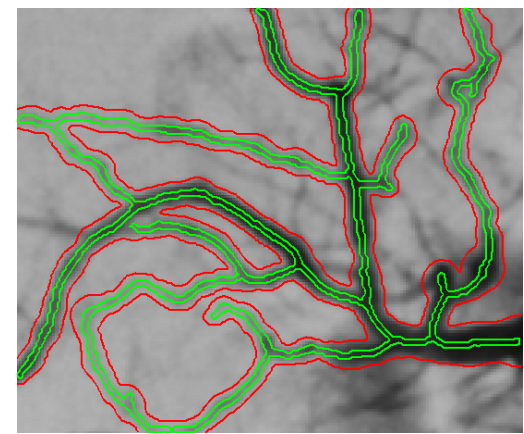

(c)

Figure 4. Results on 2D medical images with GVF-based initialization. (a) Original image. (b) Estimated centerlines and radii for initialization. (c) Final segmentation.

\subsection{Preliminary results and discussion}

Figure 4(b) depicts an example of an initial set of centerlines and radii estimation for a 2D X-ray angiography. The GVF-based initialization yields weak responses at bifurcations, especially when the intensities vary within the branches involved, which leads to disconnected centerlines. Figure 4(c) illustrates the ability of our model to propagate into bifurcations and to reconnect branches of the vascular tree. In Fig. 4(c), one can nevertheless notice how the tracking process misses a few bifurcations. This is due to the difficult trade-off between regularity and accuracy on $\sigma$. On the one hand, allowing strong and rapid variations of the radii is paramount to recover small vessels branching off from much larger arteries. Regularity and shape constraints, on the other hand, are mandatory to maintain the consistency of the model, i.e. an equivalence between $\sigma$ and the real radius of the structures. Depending on the application, one may favor one or the other and set the weights of the different constraints accordingly.

Figure 5 illustrates the above discussion with some preliminary 3D results on rotational angiographies of the brain (size $256 \times 256 \times 256,0.41 \times 0.41 \times 0.41 \mathrm{~mm}^{3}$ ). In the case of Fig. $5(\mathrm{a})$, strong constraints were applied to the model, resulting in a smooth segmentation and meaningful radii values. On the contrary, the segmentation in Fig. 5(b) was obtained using small weights for the volume constraint and the regularization over $\sigma$. Albeit more complete, the centerline of the recovered tree may spread into the whole vessels, especially into small branches (see for example the horizontal branch in the middle of Fig. 5(b) with a very ragged surface appearance due to many overlapping kernels).

Despite the challenging balance between the parameters of the model, the close-up pictures provided in Fig. $5(\mathrm{a})$ and 5(b) highlight two additional interesting features of the present work. Separating tangent vessels is often a difficult task since there is little or no contrast between the structures. Figure 5 demonstrates that our model is robust to this setting and is able to generate distinct surfaces for tangent vessels. Moreover, our model can deal with aneurysms, which are finely segmented, with a smooth transition of $\sigma$ values.

The proposed framework can be adapted to various modalities and anatomical regions. Figures 5(c) and 5 (d) show similar results for the segmentation of the portal veinous system in a 3D-CT scan of the liver (size $435 \times 435 \times 109$, spatial resolution $0.67 \times 0.67 \times 1.90 \mathrm{~mm}^{3}$ ). A mask of the liver was first computed during an additional pre-processing step to restrain the propagation to the liver. The segmentation is then performed according to the methods in Sect. 2. Because of the proximity to the hepatic tree, the weight of the regularity term on the radii map is set to a high value $(\mu=0.8)$ compared to the regularity constraint on the medialness function $(\lambda=0.1)$. As illustrated in Fig. 5(d), most branches of the portal tree are recovered during the segmentation. 


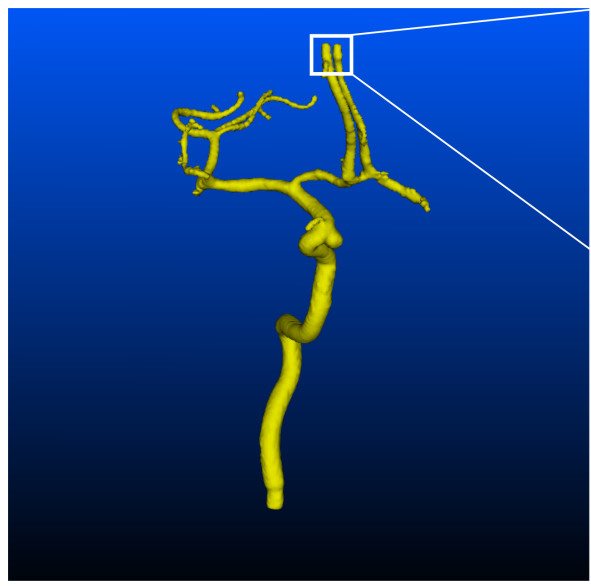

(a)

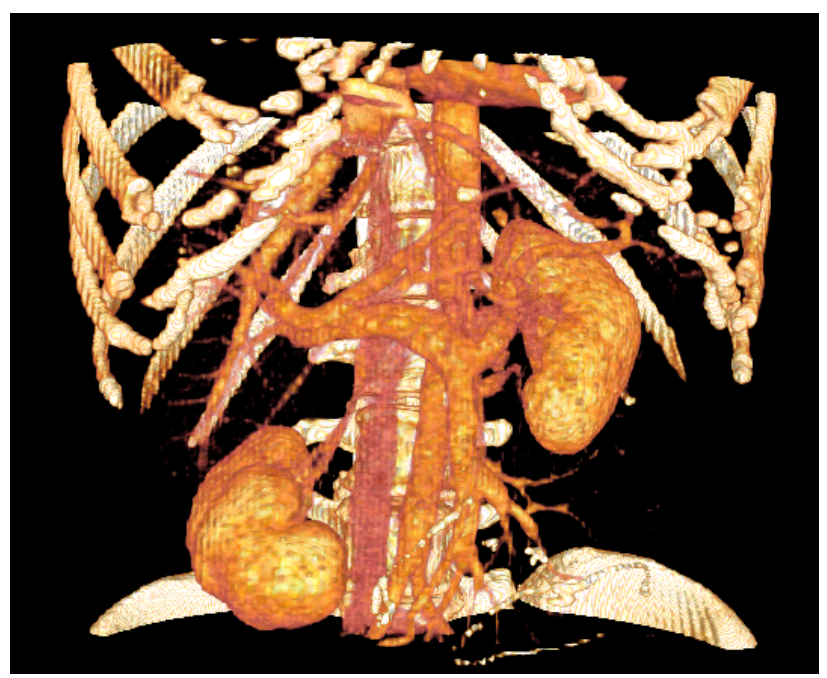

(c)
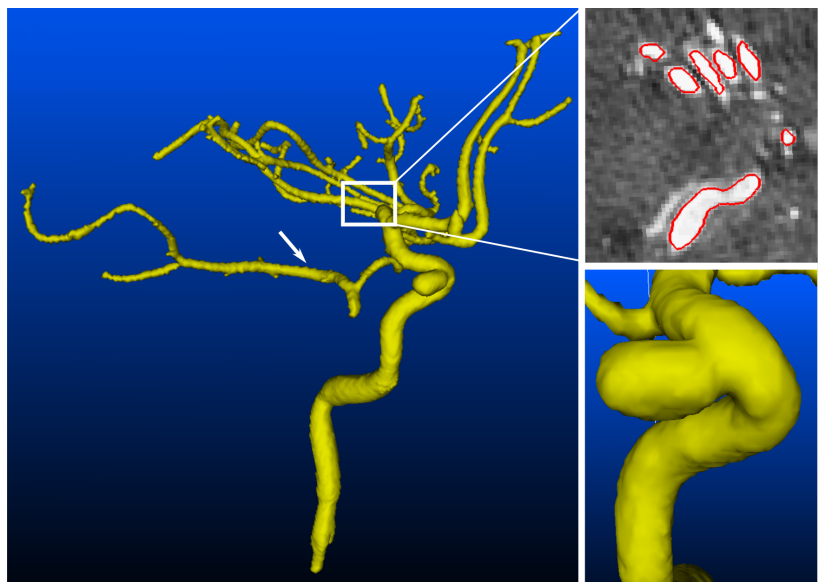

(b)

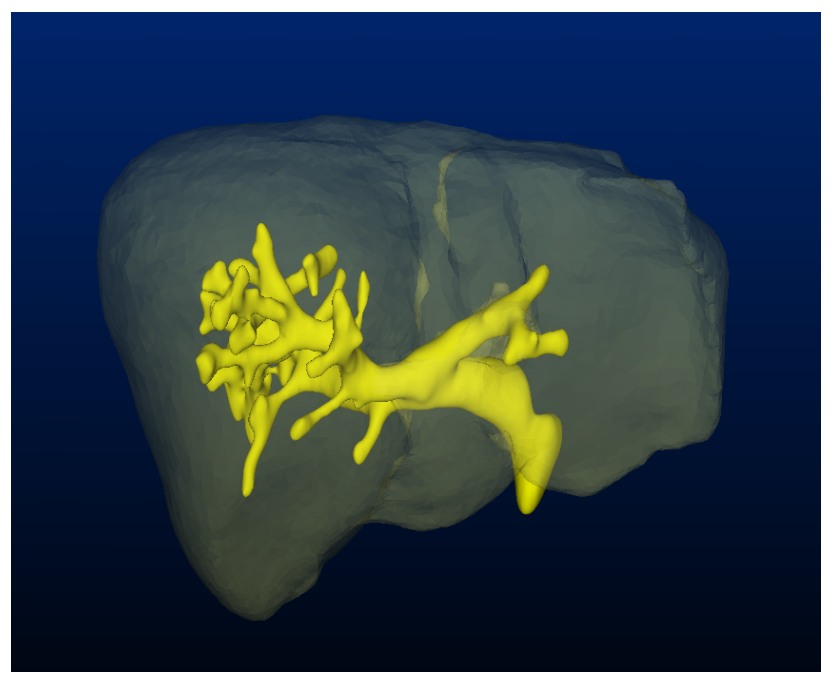

(d)

Figure 5. (a-b) Results on 3D rotational angiographies of the skull. For both segmentations of the arterial tree, we provide two close-ups to demonstrate the ability of the algorithm to separate tangent vessels (top) and to cope with aneurysms (bottom). (c-d) Segmentation of the portal tree in the liver in a 3D-CT scan (size: 435x435x109, spatial resolution: $0.67 \times 0.67 \times 1.90 \mathrm{~mm}^{3}$ ). (c) Volume rendering of the raw data. (d) Liver mask and portal veins segmented with the method described in Sect. 2.

\section{CONCLUSION}

We presented a novel region-based segmentation framework for tree-like structures, based on two coupled implicit surfaces evolving in a level set setting. This representation is able to propagate naturally through bifurcations. The use of a continuous scale parameter allows us to estimate accurately the radii of the vessels. We showed that our approach performs well in challenging configurations such as tangent vessels and aneurysms, both on synthetic and real images. Ongoing work focuses on a thorough quantitative evaluation of the segmentation results, as well as the development of a hierarchical approach to deal with a wide range of vessel radii and to reduce the influence of the regularization parameters.

\section{REFERENCES}

[1] Frangi, A.F., Niessen, W.J., Vincken, K.L. and Viergever, M.A., "Multiscale Vessel Enhancement Filtering," MICCAI 98 LNCS 1496, 130-137 (1998). 
[2] Krissian, K., Malandain, G., Ayache, N., Vaillant, R. and Trousset, Y., "Model-based Detection of Tubular Structures," Computer Vision and Image Understanding 80(2), 130-171 (2000).

[3] Friman, O., Hindennach, M. and Peitgen, H.-O., "Template-based Multiple Hypotheses Tracking of Small Vessels," Proc. ISBI 08 , 1047-1050 (2008).

[4] Lorigo, L.M., Faugeras, O.D., Grimson, W.E.L., Keriven, R., Kikinis, R. and Westin, C.-F., "Co-dimension 2 Geodesic Active Contours for MRA Segmentation," Proc. IPMI 99 , 126-139 (1999).

[5] Manniesing, R., Velthuis, B.K., van Leeuwen, M.S., van der Schaaf, I.C., van Laar, P.J. and Niessen, W.J., "Level Set Based Cerebral Vasculature Segmentation and Diameter Quantification in CT Angiography," Medical Image Analysis 10, 200-214 (2006).

[6] Florin, C., Paragios, N. and Williams, J., "Particle Filters, a Quasi-Monte Carlo Solution for Segmentation of Coronaries," MICCAI 05 LNCS 3749, 246-253 (2005).

[7] Schaap, M., Manniesing, R., Smal, I., van Walsum, T., van der Lugt, A. and Niessen, W.J., "Bayesian Tracking of Tubular Structures and Its Application to Carotid Arteries in CTA," MICCAI 07 LNCS 4792, $562-570$ (2007).

[8] Lesage, D., Angelini, E., Bloch, I. and Funka-Lea, G., "A Review of 3D Vessel Lumen Segmentation Techniques: Models, Features and Extraction Schemes," Medical Image Analysis 13(6), 819-845 (2009).

[9] Deschamps, T. and Cohen, L., "Minimal Paths in 3D Images and Application to Virtual Endoscopy," ECCV 00 LNCS 1843, 543-557 (2000).

[10] Li, H., Yezzi, A. and Cohen, L., "3D Multi-branch Tubular Surface and Centerline Extraction with 4D Iterative Key Points," MICCAI 09 LNCS 5762, 1042-1050 (2009).

[11] Oeltze, S. and Preim, B., "Visualization of Vasculature with Convolution Surfaces: Method, Validation and Evaluation," IEEE Transactions on Medical Imaging 24(4), 540-548 (2005).

[12] Lefevre, T., Mory, B., Ardon, R., Sanchez-Castro, J. and Yezzi, A., "Automatic Inferior Vena Cava Segmentation in Contrast-Enhanced CT Volumes," Proc. ISBI 10 , 420-423 (2010).

[13] Mory, B., Ardon, R. and Thiran, J.-P., "Fuzzy Region Competition: A Convex Two-Phase Segmentation Framework," Proc. SSVM 07 LNCS 4485, 214-226 (2007).

[14] Chambolle, A., "An Algorithm for Total Variation Minimization and Applications," Journal of Mathematical Imaging and Vision 20(1-2), 89-97 (2004).

[15] Nain, D., Yezzi, A. and Turk, G., "Vessel Segmentation Using a Shape Driven Flow," MICCAI 04 LNCS 3216, 51-59 (2004).

[16] Xu, C. and Prince, J.L., "Snakes, Shapes, and Gradient Vector Flow," IEEE Transactions on Image Processing 7(3), 359-369 (1998).

[17] Bauer, C. and Bischof, H., "A Novel Approach for Detection of Tubular Objects and Its Application to Medical Image Analysis," 30th DAGM Symposium on Pattern Recognition (DAGM 2008) (2008).

[18] Aylward, S.R. and Bullit, E., "Initialization, Noise, Singularities, and Scale in Height Ridge Traversal for Tubular Object Centerline Extraction," IEEE Transactions on Medical Imaging 21(2), 61-75 (2002). 